\title{
Nasolabial Cyst: Review of Literature and a Case Report
}

\author{
Paramjit Kajla $\cdot$ Jeevan Lata $\cdot$ Reecha Agrawal
}

Received: 5 February 2010/Accepted: 14 October 2010/Published online: 25 March 2011

(C) Association of Oral and Maxillofacial Surgeons of India 2011

\begin{abstract}
The nasolabial cyst is a rare non-odontogenic cyst that develops within the skin adjacent to the ala of nose around the uppermost portion of the nasolabial crease; its pathogenesis is uncertain. This lesion grows slowly and measures between 1.5 to $3.0 \mathrm{~cm}$; it is characterized clinically by a floating tumefaction in the nasolabial sulcus, which elevates the upper lip. The diagnosis is essentially based on the clinical findings. Bi-digital palpation revealed fluctulance between the floor of nasal vestibule and the gingivobuccal sulcus, which helps to confirm the diagnosis. This paper reports a case of a 35 -year-old female patient that presented a soft swelling in the right ala of the
\end{abstract}

P. Kajla $(\bowtie)$

Department of Oral and Maxillofacial Surgery,

Punjab Government Dental College and Hospital,

Amritsar, Punjab, India

e-mail: rajkajla2002@gmail.com

P. Kajla

H.No. 144, Urban Estate, Phase 1, Amritsar, Punjab, India

J. Lata

Department of Oral and Maxillofacial Surgery,

Punjab Government Dental College and Hospital,

Amritsar, Punjab 143001, India

J. Lata

15-Pink Avenue, Majitha Road, Near Madaan Hospital,

Amritsar, Punjab 143001, India

R. Agrawal

Department of Oral and Maxillofacial Surgery,

Punjab Government Dental College and Hospital,

Amritsar, Punjab 143001, India

e-mail: dr_reecha83@yahoo.co.in

R. Agrawal

Room No. 28, Girls Hostel, Govt. Dental College and Hospital,

Amritsar, Punjab 143001, India nose; the clinical features suggested a nasolabial cyst. Complete surgical excision of the cyst was done under local anesthesia and the diagnosis was confirmed by histopathology. In spite of the low occurrence of nasolabial cysts, it is important to recognize the clinical characteristics of this lesion. The purpose of this paper is to review the literature and discuss the histopathology, etiology and different treatment options of this condition.

Keywords Nasolabial cyst · Nonodontogenic cyst . Enucleation

\section{Introduction}

The nasolabial cyst is a rare non-odontogenic maxillofacial soft tissue swelling. Zuckerkandl originally described it in 1882 [1]. In 1953, Klestadt [2] investigated nasolabial cysts in depth, after which the lesion was named Klestadt's Cyst. Thoma [3] suggested the term nasoalveolar cyst, but it was Rao (1951) [4] who first used the term nasolabial cyst.

Two main etiological theories have been proposed. One holds that the lesion arises from trapped nasolacrimal duct tissue, and the other affirms that it is an embryonic fissural cyst. Klestadt [2] postulated an embryologic origin for these cysts and considered that these lesions must originate from embryonic epithelium, entrapped in the developmental fissures between the lateral nasal and maxillary processes. Since then, many authors have classified this entity based on Klestadt's embryologic theory as a fissural cyst.

Clinically, the patient had a swelling adjacent to the nose with fullness in the canine fossa and the nasal floor. The swelling causes obliteration of the nasolabial fold and elevation of the alae of the nose. It is a fluctuant swelling 
and is best palpated bimanually with one finger in the nasal floor and one finger in the labial sulcus. Nasolabial cysts are unilateral with no prevalence of side occurrence, but bilateral cases have also been reported [5]. It is lined by pseudostratified ciliated or nonciliated columnar epithelium with goblet cells. It is most commonly seen in adults, with peak prevalence in the fourth and fifth decades of life. Other significant findings include greater incidence in females (4:1) and possible greater prevalence among blacks [6].

In this report we have described a case of nasolabial cyst with its clinical features, diagnosis and treatment.

\section{Case Report}

A 35-year old female reported to our department, with a history of painless swelling in right anterior maxillary region of three years duration (Fig. 1). The swelling had been gradually increasing in size. She had right nasal obstruction of 3 months duration with no history of nasal discharge or bleeding. There was no history of trauma. She had history of extraction of maxillary central incisors in some dental clinic 3 months ago for the treatment of this swelling, which did not subside after the extraction. On examination, there was a facial asymmetry due to bulge on the right side of the nose, obstructing the right anterior nostril. The swelling was $2.0 \times 3.0 \mathrm{~cm}$ soft, fluctuating, non-tender, subcutaneous causing obliteration of the nasolabial fold. Intra-oral examination, revealed bulging of the buccoalveolar sulcus by the swelling (Fig. 2). The periapical radiograph showed no bone or dental alterations which suggested a soft tissue lesion. Making the

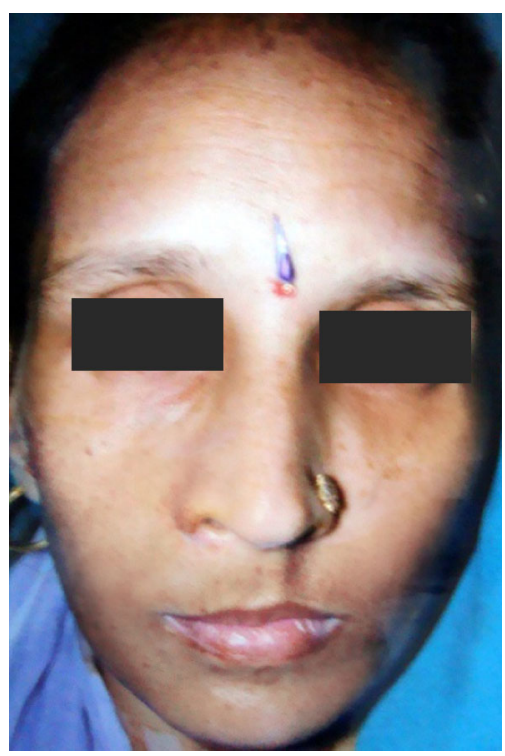

Fig. 1 Pre-operative extra-oral photograph

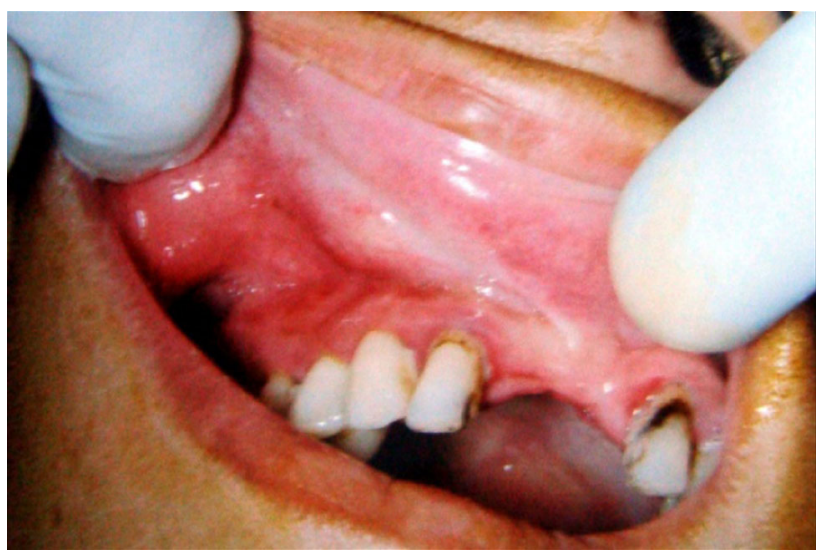

Fig. 2 Pre-operative intra-oral photograph

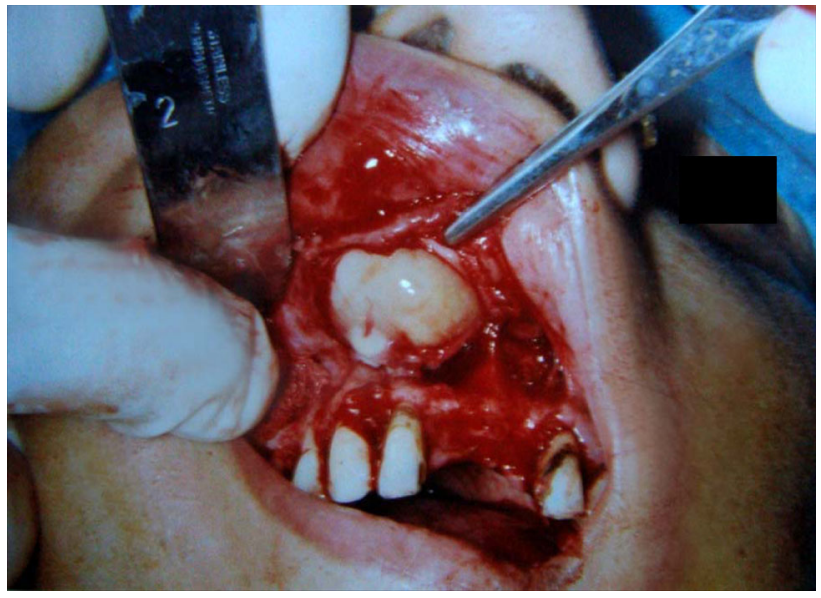

Fig. 3 Surgical exposure of the cyst through vestibular incision

provisional diagnosis of nasolabial cyst, surgical excision of the lesion was done under LA (Fig. 3) and specimen sent for biopsy.

Histopathologic findings of the excised lesion were as follows: The section of the cyst wall showed pseudostratified columnar epithelium with intermittent occurrence of goblet like mucin producing cells and also cuboidal epithelial lining. The stroma exhibited non specific chronic inflammatory infiltrate. The lesion was diagnosed by the pathologist as nasolabial cyst (Fig. 4).

The postoperative course was uncomplicated and there was no lesion recurrence up to one year of follow-up and the pathologic signs and symptoms of the lesion had disappeared (Figs. 5, 6).

\section{Discussion}

Nasolabial cysts represent about $0.7 \%$ of all cysts in the maxillofacial region, and $2.5 \%$ of non-odontogenic cysts. 


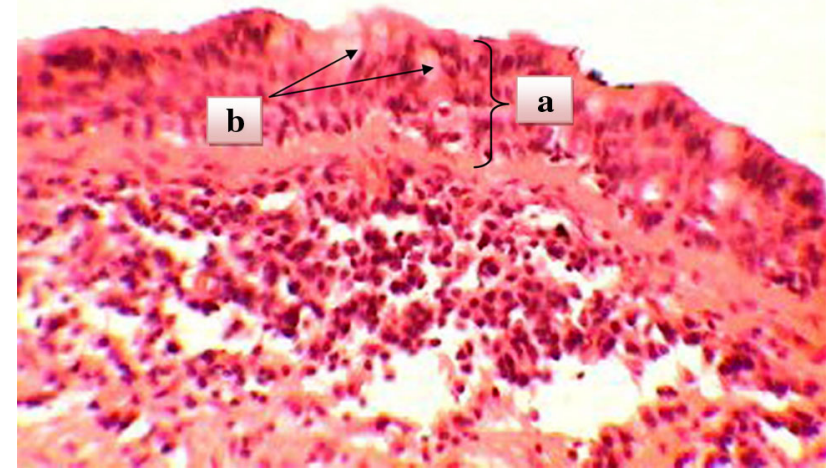

Fig. 4 Histopathological examination. a, b: Pseudostratified columnar epithelium with intermittent occurrence of goblet like mucin producing cells, stroma exhibited non specific chronic inflammatory infiltrate

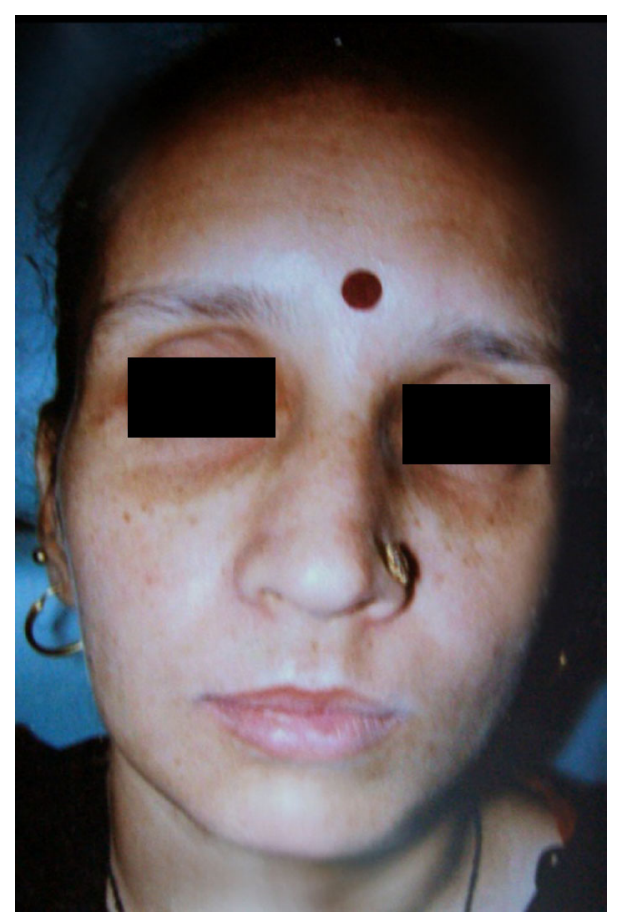

Fig. 5 Post-operative extra-oral photograph

El-Hamd reported that complications, when they occur, generally cause nasal obstruction and esthetic problems. Cohen \& Hertzanu [6] have stated that patients only seek therapy when there is deformity, nasal obstruction or infection caused by this lesion.

The diagnosis of nasolabial cysts is essentially clinical. Bidigital palpation reveals a fluctuating swelling between the floor of the nasal vestibule and the gingivolabial sulcus, which helps to confirm the diagnosis. Radiographs do not detect this soft tissue lesion except when it causes significant maxillary bone erosion. More sophisticated image diagnosis, such as computed tomography (CT) and

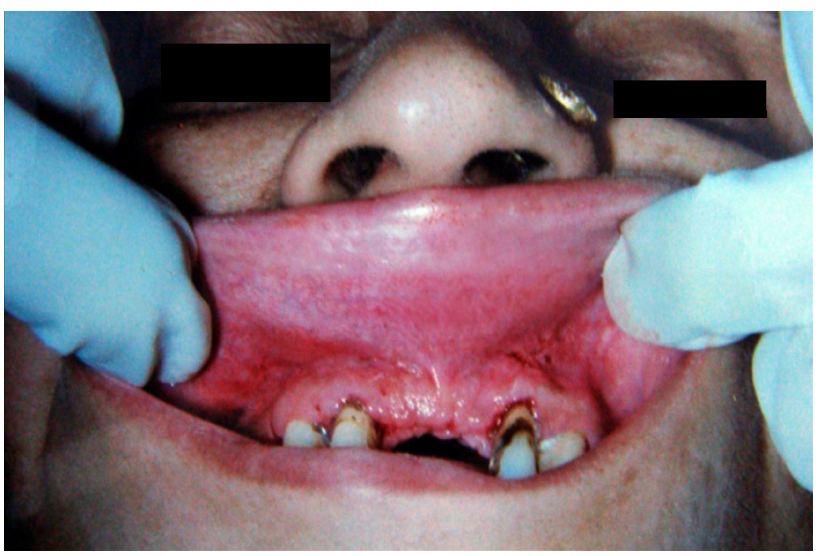

Fig. 6 Post-operative intra-oral photograph

magnetic resonance imaging (MRI), may reveal the cystic nature of these lesions in greater detail and reliability, their relation with the nasal alae and the maxillary bone, as well as bone involvement, which facilitate the diagnosis. As in our case the diagnosis was clear cut so no image examination was done.

The differential diagnosis is made with odontogenic lesions such as canine space abscess, follicular, periodontal and residual cysts, and salivary gland neoplasms [7]; only one case of carcinoma progressing from a nasolabial cyst has been described in literature. Infected nasolabial cysts may be mistaken for furuncle of the nasal vestibule floor; except for this entity, however, the features of infected nasolabial cysts are very specific, and there is little doubt in the diagnosis.

Brown-Kelly first described the histopathology of this lesion in 1898 [8]. The cyst consists of respiratory epithelium (pseudostratified ciliated cylindrical or stratified ciliated cylindrical epithelium with goblet cells), although squamous metaplasia may occur in infected cysts [9]. Fluid contained within cysts is produced by goblet cells.

Injection of sclerotic substances, marsupialization and surgical removal may be considered for treatment. However, unlike some of the large intraosseous cysts, this soft-tissue lesion does not respond to marsupialization [10] and complete surgical removal is the treatment of choice. The aim of complete excision are to prevent reoccurence, to establish a histopathological diagnosis and to ameliorate a cosmetic deformity. Care must be taken not to rupture the cyst and it should be removed intact, although there have been no reports of recurrence of these cysts after intraoperative rupture. Because this cyst is usually close related to the floor of the nose [11], perforation of the nasal mucosa may be expected during its removal. When very small perforations are caused, they can be left untreated; however, larger ones must be sutured. 
The diagnosis in this study was based on clinical findings, which described all of the findings of nasolabial cyst and was confirmed by histopathology. Also all the features of this rare entity were consistent with the literature.

\section{References}

1. Kuriloff DB (1987) The nasolabial cyst-nasal hamartoma. Otolaryngol Head Neck Surg 96(3):268-272

2. Klestadt W (1953) Nasal cysts and the facial cleft cyst theory. Annals Otol Rhinol Laryngol 62:84

3. Thoma KH (1941) Nasoalveolar cysts. Am J Orthod 27:48-52
4. Rao RV (1995) Nasolabial cyst. J Laryngol Otol 69:352-354

5. Precious DS (1987) Chronic nasolabial cyst. J Canad Dent Assoc 53:307-308

6. Cohen MA, Hertzanu Y (1985) Huge growth potential of the nasolabial cyst. Oral Surgery 59:441-445

7. El-Hamd KEAA (1999) Nasolabial cyst: a report of eight cases and a review of the literature. J Laryngol Otol 113:747-749

8. Burtschi TA, Stout RA (1963) Bilateral nasoalveolar cysts. Oral Surg 16:271-275

9. Kuriloff DB (1987) The nasolabial cyst-nasal hamartoma. Otolaryngol Head Neck Surg 96:268-272

10. Crowford W, Korchin L, Greskovich FJ (1968) Nasolabial cysts: report of two cases. J Oral Surg 26:582-588

11. Brandão GS, Ebling H, Souza IF (1974) Bilateral nasolabial cyst. Oral Surg 37:480-484 